\title{
Erratum to: The Combined Use of Environmental and Experiential Simulations to Design and Evaluate Urban Transformations
}

\author{
Barbara E.A. Piga
}

\section{Erratum to:}

Chapter 32 in: R. Cocci Grifoni et al., Quality of Life in Urban Landscapes, The Urban Book Series, https://doi.org/10.1007/978-3-319-65581-9_32

An error in the production process unfortunately led to publication of this chapter prematurely, before incorporation of the final corrections. The version supplied here has been corrected and approved by the author [authors]. 